\title{
Decreased basal and stimulated salivary parameters by histopathological lesions and secretory dysfunction of parotid and submandibular glands in rats with type 2 diabetes
}

\author{
SI-YU CHEN $^{1 *}$, YING WANG $^{1 *}$, CHUN-LEI ZHANG ${ }^{2 *}$ and ZE-MIN YANG ${ }^{1}$ \\ ${ }^{1}$ Department of Biochemistry and Molecular Biology, School of Life Sciences and Biopharmaceutics, \\ Guangdong Pharmaceutical University, Guangzhou, Guangdong 510006; ' Department of Stomatology, \\ The First Affiliated Hospital of Jinan University, Guangzhou, Guangdong 510632, P.R. China
}

Received May 24, 2019; Accepted January 17, 2020

DOI: $10.3892 /$ etm.2020.8505

\begin{abstract}
Patients with type 2 diabetes mellitus (T2DM) present with dry mouth, polydipsia and taste impairment due to salivary secretion disorder. However, the underlying functional mechanism of T2DM remains unknown. The present study found that T2DM rats had significantly lower salivary flow rate and salivary alpha amylase activity, and attenuated salivary secretion responses to acid stimulation compared with control rats. Histopathological observation found that T2DM rats had inflammatory cell infiltration with increased expressions of IL- 6 and TNF- $\alpha$, oxidative stress, including decreased total superoxide dismutase activity and increased malondialdehyde content, and decreased expressions of $\beta 1$ adrenergic receptor, cholinergic receptor, aquaporin-5 and protein kinase A in salivary glands, in particular the parotid gland. These results indicated that parotid gland impairment was more severe compared with submandibular gland impairment. Reduced salivary secretion may be associated with histopathological lesions and decreased regulation in secretory pathways in salivary glands.
\end{abstract}

Correspondence to: Dr Ze-Min Yang, Department of Biochemistry and Molecular Biology, School of Life Sciences and Biopharmaceutics, Guangdong Pharmaceutical University, 280 Waihuan Road East, Guangzhou, Guangdong 510006, P.R. China

E-mail: yzm3102001@gmail.com; 99394722@qq.com

*Contributed equally

Abbreviations: T2DM, type 2 diabetes mellitus; sAA, salivary alpha amylase; $\beta 1-\mathrm{AR}, \beta 1$ adrenergic receptor; $\mathrm{M} 3 \mathrm{R}$, cholinergic receptor; AQP5, aquaporin-5; Gs, adenylate cyclase; AC, adenylate cyclase; cAMP, cyclic adenosine monophosphate; PKA, protein kinase A; PLC- $\beta$, phospholipase C- $\beta$; IP3, inositol 1,4,5-trisphosphate; T-SOD, total superoxide dismutase; MDA, malondialdehyde

Key words: type 2 diabetes mellitus, parotid glands, submandibular gland, salivary parameters, histopathological lesion, secretory dysfunction

\section{Introduction}

Type 2 diabetes mellitus (T2DM) is a metabolic disease characterized by hyperglycemia. T2DM can lead to serious complications, including cardiovascular disease, blindness, renal failure and amputation. The pathogenesis of T2DM is complex and oxidative stress, in addition to inflammation are two crucial risk factors. Metabolic disorders may lead to oxidative stress by generating the reactive oxygen species (ROS). ROS could activate nuclear factor- $\kappa \mathrm{B}$ pathway and produce various proinflammatory cytokines. These factors could deteriorate the islets $\beta$-cells of the pancreas and insulin signaling pathways, resulting in reduced release of insulin and insulin resistance (1). Meanwhile, persistent hyperglycaemia in T2DM also increases the production of ROS and inflammatory cytokines, damaging various organ systems and leading to diabetic complications. In addition, clinical observations have indicated that patients with T2DM have typical symptoms, including dry mouth and polydipsia, even taste impairment. Therefore, taking the aforementioned into consideration, it is suggested that hyperglycemia may cause salivary secretion disorder in patients with T2DM by oxidative stress and inflammation.

Saliva is mainly secreted by the parotid gland, the submandibular gland and the sublingual gland. The submandibular gland serves an role in basal salivary secretion, while the parotid gland serves an important role in stimulated salivary secretion. However, the sublingual gland serves an minor role in salivary secretion. Salivary secretion is mainly regulated by the autonomic nervous system. The sympathetic nerve regulates the secretion of a large number of protein saliva, including salivary alpha-amylase (sAA) by beta adrenergic receptors $(\beta-A R) / G$ protein stimulating adenylate cyclase (Gs)/adenylate cyclase (AC)/cyclic adenosine monophosphate (cAMP)/protein kinase A (PKA) signaling pathway; The parasympathetic nerve regulates the secretion of a large number of fluid saliva, such as electrolytes, water and a little salivary proteins by choline receptor $(\mathrm{M} 3 \mathrm{R}) / \mathrm{G}$ protein subunit alpha Q (Gq)/phospholipase C- $\beta$ (PLC- $\beta$ )/inositol 1,4,5-trisphosphate (IP3)/ $\mathrm{Ca}^{2+} /$ aquaporin-5 (AQP5) signaling pathway (2). Therefore, the function of salivary glands and the 
salivary secretion pathways are the main factors that affect the synthesis and secretion of saliva.

At present, studies on salivary secretion in patients with T2DM have mainly focused on the comparison of salivary indicators between diabetic and non-diabetic individuals, due to the limitation of clinical research. Several studies have shown that diabetic patients and healthy individuals had significant differences in the salivary parameters, including salivary glucose, sAA, s-IgA, sialic acid, total salivary protein and ion concentration (3-7), and diabetic patients exhibited pathological changes in the autonomic nervous system (8). Research on salivary secretion in diabetic animal model has been focused on pathological observation and associated mechanisms of salivary glands, due to the technological difficulty of salivary acquisition. A number of studies have reported that salivary hypofunction is associated with atrophy of the submandibular and parotid glands in diabetic rat and hamsters $(9,10)$. In addition, High et al (11) reported that the submandibular gland hypofunction is associated with the abnormity of calcium signaling pathway in diabetic rats induced by streptozotocin.

Although basal salivary secretion in T2DM had been reported in a number of studies, certain limitations remain, such as that the salivary parameters were mainly from basal saliva, and the lack of stimulated saliva; In addition, studies on salivary glands' function are mainly focused on pathological observation, and the lack of mechanism studies in inflammation and salivary secretion pathway; In particular, the association between T2DM, basal and stimulated salivary parameters, and salivary glands function remains unclear. Therefore, the present study investigated the differences in salivary secretion in basal and stimulated conditions between T2DM and control rats. The aim of the present study was to further examine the underlying mechanism of the aforementional looking at the histopathology and the expression levels of salivary secretion proteins regulated by autonomic nerves in the parotid and submandibular glands.

\section{Materials and methods}

Experimental animals. Male Sprague-Dawley (SD) rats (195-200 g) were provided by the Experimental Animal Center of Guangzhou Traditional Chinese Medicine University, and production license number was SCXK (Guangdong) 2013-0034. SD rats were bred in in the Experimental Animal Center of Guangdong Pharmaceutical University, and use license number was SYXK (Guangdong) 2017-0125. Housing conditions were controlled at $20 \pm 2^{\circ} \mathrm{C}$ at room temperature with a 12/12 h light/dark cycle. The present study was approved by the Academic Ethics Committee of Guangdong Pharmaceutical University (approval no. gdpu2016074).

Induction of T2DM rats. Once adaptive feeding patterns were established (3 days), sixteen 8-week-old SD rats were randomly divided into control and T2DM groups. Rats in the control group $(n=8)$ were kept on a basic diet, provided by the Experimental Animal Center of Guangdong Pharmaceutical University, where total energy was $3.85 \mathrm{kcal} / \mathrm{g}$, and the energy supply ratio of fat was $10 \%$. Rats in the T2DM group $(n=8)$ received a high-fat diet (HFD), provided by Guangdong Medical Experimental Animal Center, where total energy was
$4.73 \mathrm{Kcal} / \mathrm{g}$, and the energy supply ratio of fat was $45 \%$. After 8 weeks of feeding, according to preliminary experiments and references (12), rats in the T2DM group were intraperitoneally injected with $35 \mathrm{mg} / \mathrm{kg}$ streptozotocin (STZ, McLean, Shanghai, China) subsequent to fasting $>12 \mathrm{~h}$. STZ was dissolved in $0.1 \mathrm{M}$ citrate buffer at $\mathrm{pH}=4.2-4.5$ prior to use. Meanwhile, rats in the control group were injected with equal volume of citrate buffer. After one weeks, approximately $500 \mu \mathrm{l}$ blood samples from the orbital cavity of experimental rats were collected to determine the glucose blood level and insulin concentration, using the glucose oxidase method with Blood Sugar kit (cat. no. 141517011, Mindray) and the ELISA method with Insulin Detection kit (cat. no. CBS-E05070R, CUSABIO), respectively, according to the manufacture's protocols. Rats with fasting blood glucose levels with a value $\geq 11.1 \mathrm{mmol} / \mathrm{l}$, insulin resistance, and exhibiting typical symptoms including polydipsia, polyphagia, polyuria and weight loss (Table I), were considered the optimal group and therefore, were selected as T2DM group. The polydipsia and polyphagia were assessed by water and food intakes, while the polyuria was assessed by moisture level of padding. Subsequently, rats in the control group and the T2DM group were kept on their basic diet and high-fat/high-sugar diet, respectively.

Collection and processing of samples. Saliva was collected according to our previous research $(13,14)$. The protocol was as follows: Rats were fasted overnight and the experiment was carried out the following day between 9:00-11:00 am. The rats were anesthetized with $30 \mathrm{mg} / \mathrm{kg}$ pentobarbital sodium (Beijing Chemical Reagent Company) and their limbs were fixed. Basal saliva of rats (prior to stimulation) was collected for 3 min with a micro-sampler. Stimulated saliva (following stimulation) was collected by the aforementioned method. Pieces of filter paper $(0.5 \times 0.5 \mathrm{~cm})$ were soaked in $0.4 \mathrm{~mol} / 1$ citric acid solution for $10 \mathrm{~min}$, and were subsequently air dried. The aforementioned was used to stimulate the tip of the rats' tongue for $30 \mathrm{sec}$ every $2.5 \mathrm{~min}$ and saliva was collected for 5 min using a micro-sampler. The collected saliva samples were centrifuged at $10,000 \mathrm{x}$ g at $4{ }^{\circ} \mathrm{C}$ for $5 \mathrm{~min}$, and the supernatant was stored at $-20^{\circ} \mathrm{C}$.

At the end of the experiment, experimental rats were anaesthetized by intraperitoneal injection of $10 \%$ chloral hydrate $(300 \mathrm{mg} / \mathrm{kg})$. After anaesthesia was determined by pain reflex and cornea reflex, the blood of rats, more than $5 \mathrm{ml}$, was drained from abdominal aorta. After confirming that the rat heartbeat stopped for $5 \mathrm{~min}$ by touch and stethoscope, rat's parotid and submandibular glands were taken in $15 \mathrm{~min}$. Moreover, experimental animals did not exhibit signs of peritonitis after the chloral hydrate administration. These tissue specimens were washed with physiological saline, and dried with filter paper. Some of them were fixed in $4 \%$ paraformaldehyde to perform hematoxylin and eosin (H\&E) staining, and the remaining tissues were store in $-80^{\circ} \mathrm{C}$, in order to evaluate biochemical parameters determination and perform real-time quantitative PCR (RT-qPCR).

Examination of salivary parameters. The activity of sAA $(\mathrm{U} / \mathrm{ml})$ was determined using the kinetic reaction assay wiht a commercially available AMY kit (cat. no. 142817005, Mindray) on the BS-180 automatic biochemical analyzer 
(Mindray, Shenzhen, China). Briefly, chromogenic substrate, 4,6-ethylidene-G7-Q-nitrophenol, is cleaved by sAA and auxiliary enzyme $\alpha$-glucosidase into $Q$-nitrophenol (PNP). The sAA activity was determined using the PNP absorption spectrum at $405 \mathrm{~nm}$. Total protein concentration $(\mathrm{mg} / \mathrm{ml})$ was determined using the bicinchoninic acid (BCA) Protein Assay Kit (cat. no. P0011, Beyotime), and bovine serum albumin was used to obtain standard curves. All operations were strictly carried out according to the reagents manual and instrument operation instructions. Salivary flow rate was calculated by the total volume of saliva $(\mathrm{ml})$ and saliva collection time (min), which was expressed as $\mathrm{ml} / \mathrm{min}$. Salivary protein secretion rate was calculated by the total protein concentration $(\mathrm{mg} / \mathrm{ml})$ and the salivary flow rate $(\mathrm{ml} / \mathrm{min})$, which expressed as $\mathrm{mg} / \mathrm{min}$. The sAA specific activity was calculated by the sAA activity $(\mathrm{U} / \mathrm{ml})$ and the total protein concentration $(\mathrm{mg} / \mathrm{ml})$, which was expressed as $\mathrm{U} / \mathrm{mg}$. The delta $(\Delta)$ value of all salivary parameters was calculated by the parameters values prior and following acid stimulation. Salivary $\mathrm{pH}$ value was determined by precision test paper of pH 6.4-8.0 and 8.0-10.0 (Fuyang special paper Co., Ltd.).

Biochemical parameters in tissue evaluation. The parotid and submandibular glands stored at $-80^{\circ} \mathrm{C}$ were taken out to prepare into $10 \%$ tissue homogenate with pre-cooling saline using an electric homogenizer. After $10 \mathrm{~min}$ of centrifugation at $10,000 \mathrm{xg}$ at $4^{\circ} \mathrm{C}$, the supernatant was used to determine the activities of sAA and total superoxide dismutase (T-SOD, item number: A001-1, Jiancheng institute of bioengineering, Nanjing, China), and the contents of total protein, total cholesterol (TC, lot number: 141617008), triglyceride (TG, lot number: 141717008), glucose (lot number: 141517011), all purchased from Mindray, and malondialdehyde (MDA, item number: A003-1, Jiancheng Institute of bioengineering, Nanjing, China) using the BS-180 automatic biochemical analyzer. Determination of total protein content and sAA activity was described above. T-SOD activity was determined using the xanthine oxidase method. MDA content was determined using colour reaction of MDA and thibabituric acid. TC and TG contents were determined using the methods of cholesterol oxidase and glycerol-3-phosphate oxidase, respectively.

$H \& E$ staining in histopathology. Due to the fact that the sublingual gland accounts for a small proportion of salivary secretion, the focus of this study was on the other two glands, including the parotid and submandibular glands. Tissue specimens from the parotid and submandibular glands were obtained from $4 \%$ paraformaldehyde, and transferred to $70 \%$ ethanol. Tissue specimens were subsequently placed in processing cassettes to dehydrate using gradient alcohol series, and embedded in paraffin wax blocks. Prior to immunostaining, tissue slices (thickness, $2 \mu \mathrm{m}$ ) were dewaxed by xylene, rehydrated through decreasing concentrations of ethanol, and washed using phosphate buffer solution. After H\&E staining, tissue specimens were dehydrated through increasing concentrations of ethanol and xylene. Finally, histopathological characteristics of the two glands were observed and images were obtained by ZEISS microscope (Germany, magnification, $\mathrm{x} 400$ ). 
$R T-q P C R$. Total RNA from the parotid and submandibular glands was extracted by TRNzol Universal Reagent (Tiangen Biotech Co., Ltd.). The cDNA was synthesized using cDNA first chain premix reagent (directory number: KR118, Tiangen Biotech Co., Ltd.). RT-qPCR experiment was performed using the Talent fluorescence quantitative detection kit SYBR Green (directory number: FP209. Tiangen Biotech Co., Ltd., Beijing, China) to determine the expression levels of TNF- $\alpha$, IL-6, $\beta 1-\mathrm{AR}, \mathrm{M} 3 \mathrm{R}, \mathrm{AQP5}$ and PKA genes. The primers and product sizes of these genes were presented in Table II. The PCR reaction procedure was as follows: Initial denaturation of $95^{\circ} \mathrm{C}$ for $3 \mathrm{~min}$, followed by 45 cycles of $95^{\circ} \mathrm{C}$ for $5 \mathrm{sec}, 60^{\circ} \mathrm{C}$ for $10 \mathrm{sec}$ and $72^{\circ} \mathrm{C}$ for $15 \mathrm{sec}$. The $\beta$-actin gene was used as an internal control to normalize the amount of candidate genes. RT-qPCR was performed on CFX96 Real-Time PCR Detection System (Bio-Rad, California, USA) and the results were analyzed by the $2^{-\Delta \Delta c t}$ method using CFX manager software version 3.1.

Western blot analysis. The parotid and submandibular gland tissue were homogenized in RIPA lysis buffer and $1 \mathrm{mM}$ PMSF (phenylmethylsulfonyl fluoride) on ice for $30 \mathrm{~min}$, and subsequently centrifuged for $10 \mathrm{~min}$ at $10,000 \mathrm{x} \mathrm{g}$ and $4^{\circ} \mathrm{C}$ in order to extract proteins. The protein contents were determined using nucleic acid protein detector. The protein samples were boiled after adding $4 \mathrm{x}$ loading buffer for $10 \mathrm{~min}$ at $100^{\circ} \mathrm{C}$ and the protein samples of equal quantity were subsequently separated using SDS-PAGE. Proteins with different molecular weights were separated on $10 \%$ separation gel and 5\% stacking gel. The separated proteins were subsequently transferred onto polyvinylidene difluoride membranes, which were already soaked in methanol for $15 \mathrm{~min}$, and blocked with 5\% skim milk prepared with PBST (ratio of PBS and Tween-20 was 2,000:1) for $2 \mathrm{~h}$. The protein blots were subsequently incubated with primary antibodies for sAA (ab139994, Abcam, dilution, 1:1,000), $\beta$-actin (20536-1-AP, Proteintech Group, Inc., dilution, 1:5,000) and $\beta 1-\mathrm{AR}(\mathrm{ab} 3442$, Abcam, dilution, 1:1,000) overnight at $4{ }^{\circ} \mathrm{C}$. Proteins were then incubated with the corresponding Horseradish Peroxidase-conjugated Affinipure Goat Anti-Rabbit IgG (E030120-01, EarthOx Life Sciences, dilution, 1:10,000) for $2 \mathrm{~h}$ after washing with PBST in triplicate. The protein blots were stained using ECL luminous solution. Due to the difference of expression abundance, the interest and control proteins were run on different membranes. Protein bands were obtained using GeneGnome XQR gel imaging systems (Syngene) and quantified by ImageJ software (National Institutes of Health).

Statistical analysis. Prior to statistical analyses, all data were tested for normal distribution by the Kolmogorov-Smirnov test. These analyses revealed that only salivary flow rate conformed to normal distribution in control and T2DM groups. Log transformation was used for the data that did not conform to normal distribution. Students' paired t-test was used to compare the means of salivary parameters between basal and stimulated saliva, and Students' unpaired t-test was used to compare the means of salivary and tissue biochemical parameters between the two groups. Pearson's correlation analysis was used to determine the correlation between parameters in saliva and tissue. Data analysis was performed using SPSS 17.0 software (IBM Corporation Inc.). All data were

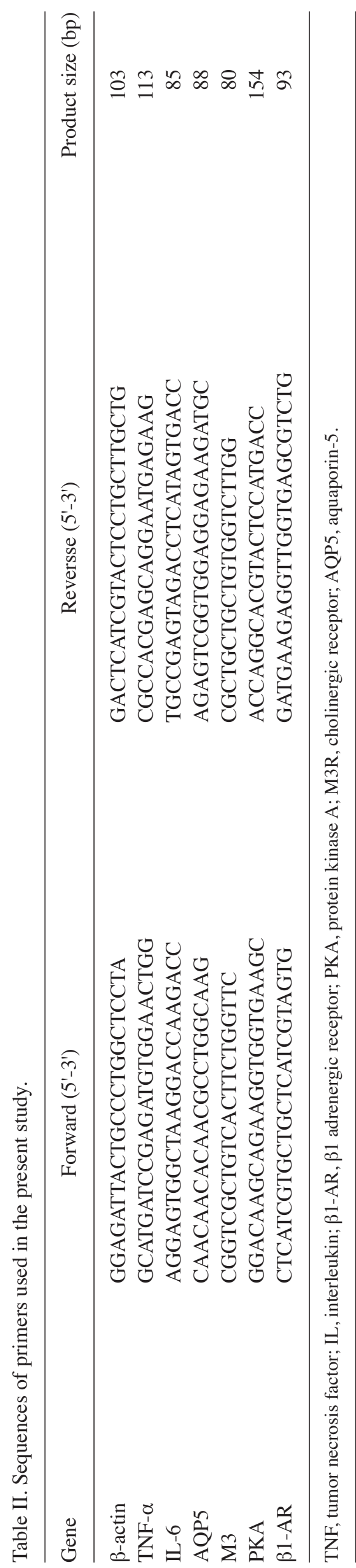



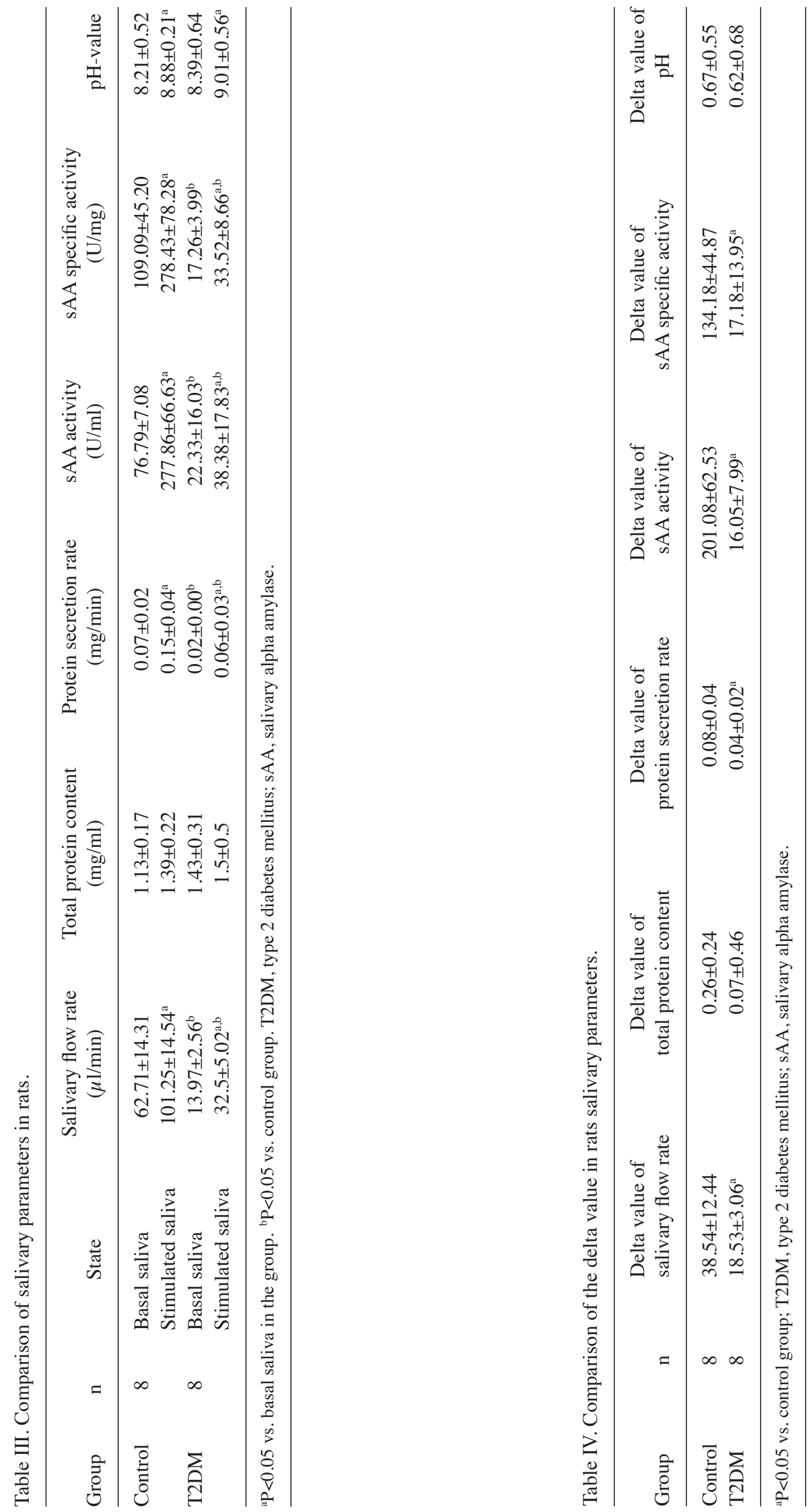
presented as mean \pm standard error. Figures were prepared using GraphPad Prism 5.0 software (GraphPad Software Inc.), $\mathrm{P}<0.05$ was considered to be indicate a statistically significant difference.

\section{Results}

General characteristics. In the initial stage of the experiment, no significant differences in body weight, water, food and engergy intakes, and fasting blood glucose concentration, were found between T2DM and control rats. After 3 weeks for STZ injection, T2DM rats showed higher intakes of water, food and energy, and decreased body weight compared with control rats. In addition, T2DM rats' padding was wetter than control rats. Serum indicators showed that T2DM rats had significantly increased fasting blood glucose concentration and HOMA-IR index, and decreased insulin levels (Table I). All these results indicated that T2DM rat model had been successfully constructed.

Salivary parameters prior and following acid stimulation. To confirm whether there was an abnormal salivary secretion in T2DM rats, salivary parameters were determined prior and following acid stimulation for control and T2DM rats. The results were showed in Tables III and IV. For the basal and stimulated salivary parameters (Table III), T2DM rats showed significantly lower salivary flow rate $\left(\mathrm{P}_{\text {basal }}=0.0005\right.$; $\left.\mathrm{P}_{\text {stimulated }}=0.0006\right)$, protein secretion rate $\left(\mathrm{P}_{\text {basal }}=0.0042\right.$; $\left.\mathrm{P}_{\text {stimulated }}=0.010\right)$, sAA activity $\left(\mathrm{P}_{\text {basal }}=0.0003 ; \mathrm{P}_{\text {stimulated }}=0.0003\right)$ and sAA specific activity $\left(\mathrm{P}_{\text {basal }}=0.0005 ; \mathrm{P}_{\text {stimulated }}=0.0007\right)$ compared with control rats. For salivary parameters responses to acid stimulation (Table III), T2DM and control rats showed significant increase in salivary flow rate $\left(\mathrm{P}_{\mathrm{T} 2 \mathrm{DM}}\right.$ $\left.<0.001 ; \mathrm{P}_{\text {control }}=0.006\right)$, protein secretion rate $\left(\mathrm{P}_{\mathrm{T} 2 \mathrm{DM}}=0.014\right.$; $\left.\mathrm{P}_{\text {control }}=0.024\right)$, sAA activity $\left(\mathrm{P}_{\mathrm{T} 2 \mathrm{DM}}=0.005 ; \mathrm{P}_{\text {control }}=0.001\right)$ and sAA specific activity $\left(\mathrm{P}_{\mathrm{T} 2 \mathrm{DM}}=0.031 ; \mathrm{P}_{\text {control }}=0.022\right)$, and $\mathrm{pH}$ value $\left(\mathrm{P}_{\mathrm{T} 2 \mathrm{DM}}=0.011 ; \mathrm{P}_{\text {control }}<0.001\right)$ after the acid stimulation. However, T2DM rats showed lower delta values in salivary flow rate $(\mathrm{P}=0.0276)$, protein secretion rate $(\mathrm{P}=0.0500)$, $\mathrm{sAA}$ activity $(\mathrm{P}=0.0158)$ and $\mathrm{sAA}$ specific activity $(\mathrm{P}=0.0353)$ compared with control rats (Table IV). In addition, no significant differences in total protein concentration were found between the two groups.

Biochemical parameter observation in salivary glands. In order to further confirm the histopathological lesions of salivary glands, the biochemical parameters from salivary glands were determined. The results (Table V) showed that, compared with control rats, T2DM rats had significantly higher glucose $\left(\mathrm{P}_{\text {parotid gland }}<0.001 ; \mathrm{P}_{\text {submandibular gland }}=0.011\right)$ content, and significantly lower sAA $\left(\mathrm{P}_{\text {parotid gland }}=0.025 ; \mathrm{P}_{\text {submandibular gland }}=0.050\right)$ and T-SOD activities ( $\mathrm{P}_{\text {parotid gland }}=0.022 ; \mathrm{P}_{\text {submandibular gland }}$ $<0.001)$ in parotid and submandibular glands. In addition, the contents of TC $\left(\mathrm{P}_{\text {parotid gland }}=0.003 ; \mathrm{P}_{\text {submandibular gland }}=0.176\right)$ and MDA $\left(\mathrm{P}_{\text {parotid gland }}<0.001 ; \mathrm{P}_{\text {submandibular gland }}=0.625\right)$ in T2DM rats' parotid gland, and total protein contents $\left(\mathrm{P}_{\text {parotid gland }}=0.664\right.$; $\mathrm{P}_{\text {submandibular gland }}=0.017$ ) in T2DM rats' submandibular gland, was significantly higher compared with control rats. No significant differences in TG content of parotid and submandibular glands were found between control and T2DM rats $(\mathrm{P}>0.05)$. 
A

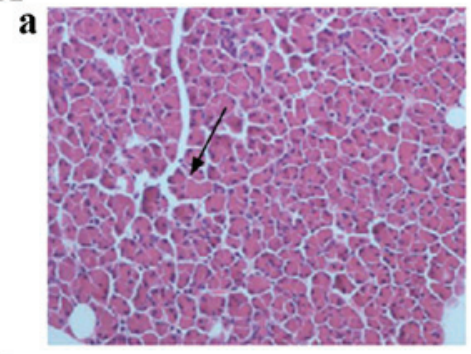

B

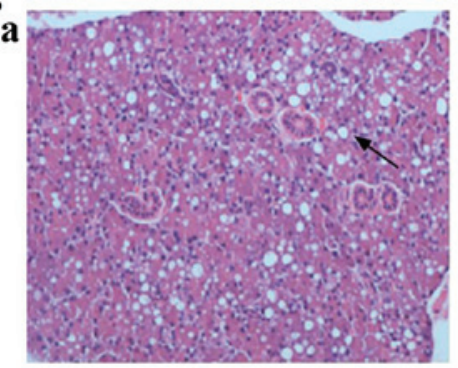

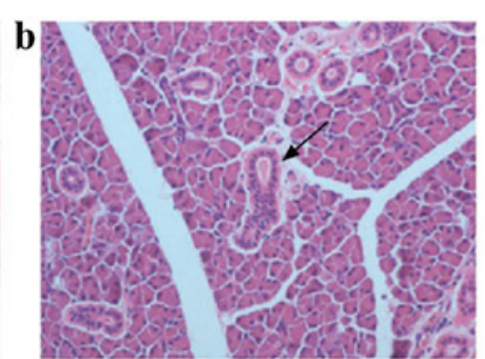

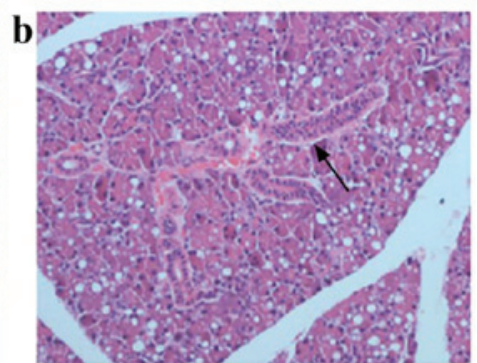

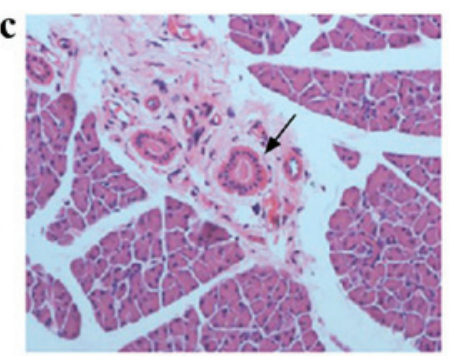

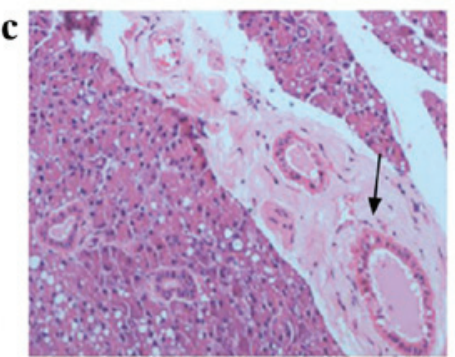

Figure 1. Histopathomorphology of the parotid gland of rats (H\&E; magnification, x400). Parotid gland tissue in control rats was able to be observed in the acinar cells with a regular shape (arrow in A-a), the glandular ducts (arrow in A-b) and their lumen (arrow in A-c) with an orderly arrangement of the parietal cells, respectively. Parotid gland tissue in T2DM rats were able to be observed and exhibited vacuolization of the acinar cells (arrow in B-a), the glandular ducts with disorderly arrangement of the parietal cells (arrow in B-b), and glandular lumen accompanied by dilation and inflammatory cell infiltration (arrow in B-c).

A
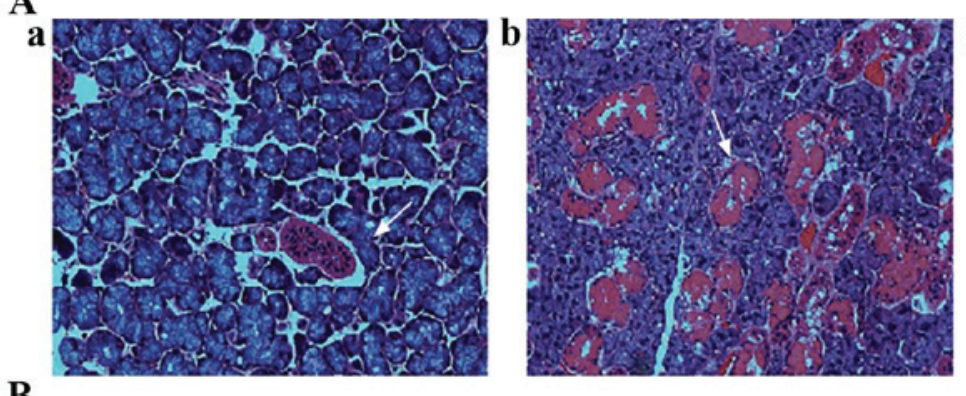

B
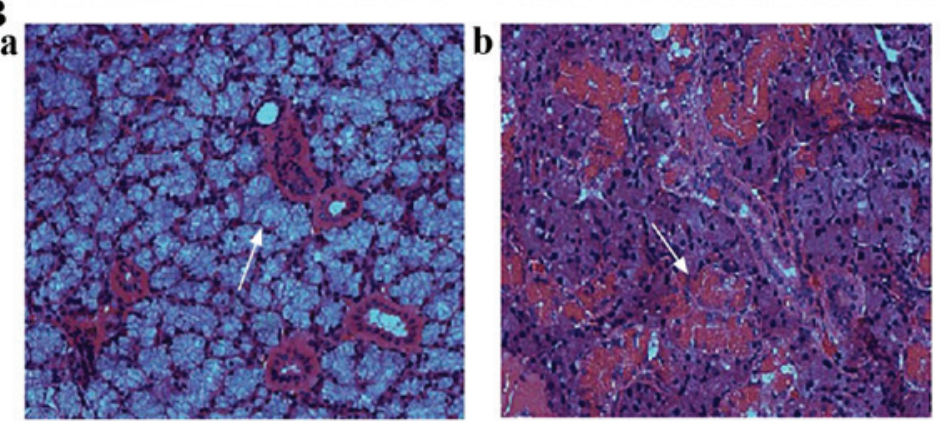
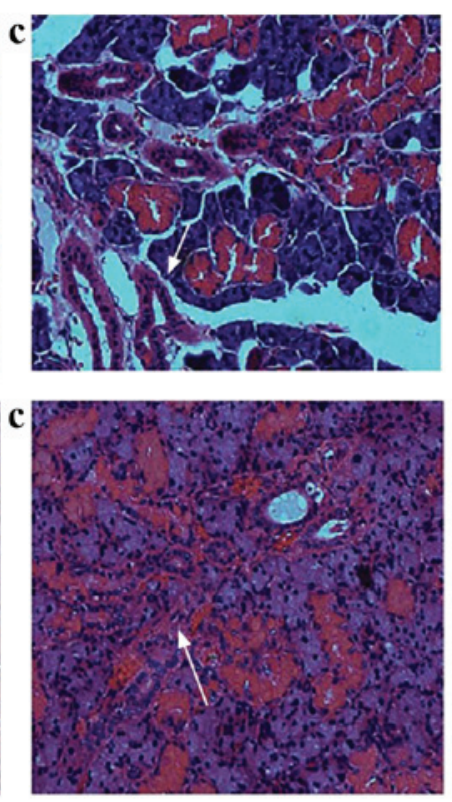

Figure 2. Histopathomorphology of the submandibular gland of rats (H\&E; magnification, x400). Submandibular gland tissue in control rats were able to be observed the serous acinar cell (arrow in A-a) and the mucous acinar cell (arrow in A-b) with regular shape. The lumen of the glandular duct was clear, and the parietal cells were arranged neatly, surrounded by fibrous tissue (arrow in A-c). Submandibular gland tissue in T2DM rats were able to be observed in the serous acinar cells and were indicated to have disappeared and formed adhesion, with blurred cytoplasm and basal stria (arrow in B-a). No stopathological lesions were observed in the glandular ducts and mucous acinar cells (arrow in B-b and -c).

Correlations between biochemical parameters in saliva and tissue. Basal and stimulated salivary flow rate $\left(\mathrm{R}_{\text {basal }}=-0.532\right.$, $\left.\mathrm{P}=0.005, \mathrm{R}_{\text {stimulatted }}=-0.554, \mathrm{P}=0.003\right)$, and stimulated sAA activity $\left(\mathrm{R}_{\text {stimulatted }}=-0.375, \mathrm{P}=0.059\right)$, were negatively correlated with fasting blood glucose concentration. In parotid and submandibular glands, there were a negative correlations between sAA activity and glucose concentration $\left(\mathrm{R}_{\text {parotid }}=-0.854, \mathrm{P}=0.030, \mathrm{R}_{\text {submandibular }}=-0.805, \mathrm{P}=0.054\right)$, between T-SOD activity and protein content $\left(\mathrm{R}_{\text {parotide }}=0.920\right.$, $\left.\mathrm{P}=0.009, \mathrm{R}_{\text {submandibular }}=-0.980, \mathrm{P}=0.001\right)$. In addition, TC content was positively correlated with glucose concentration in parotid gland $(\mathrm{R}=0.837$ and $\mathrm{P}=0.038)$. No significant correlations were found between the others biochemical parameters.

Histopathological observation of salivary glands. In order to analyze the cause of disturbance in salivary secretion in 

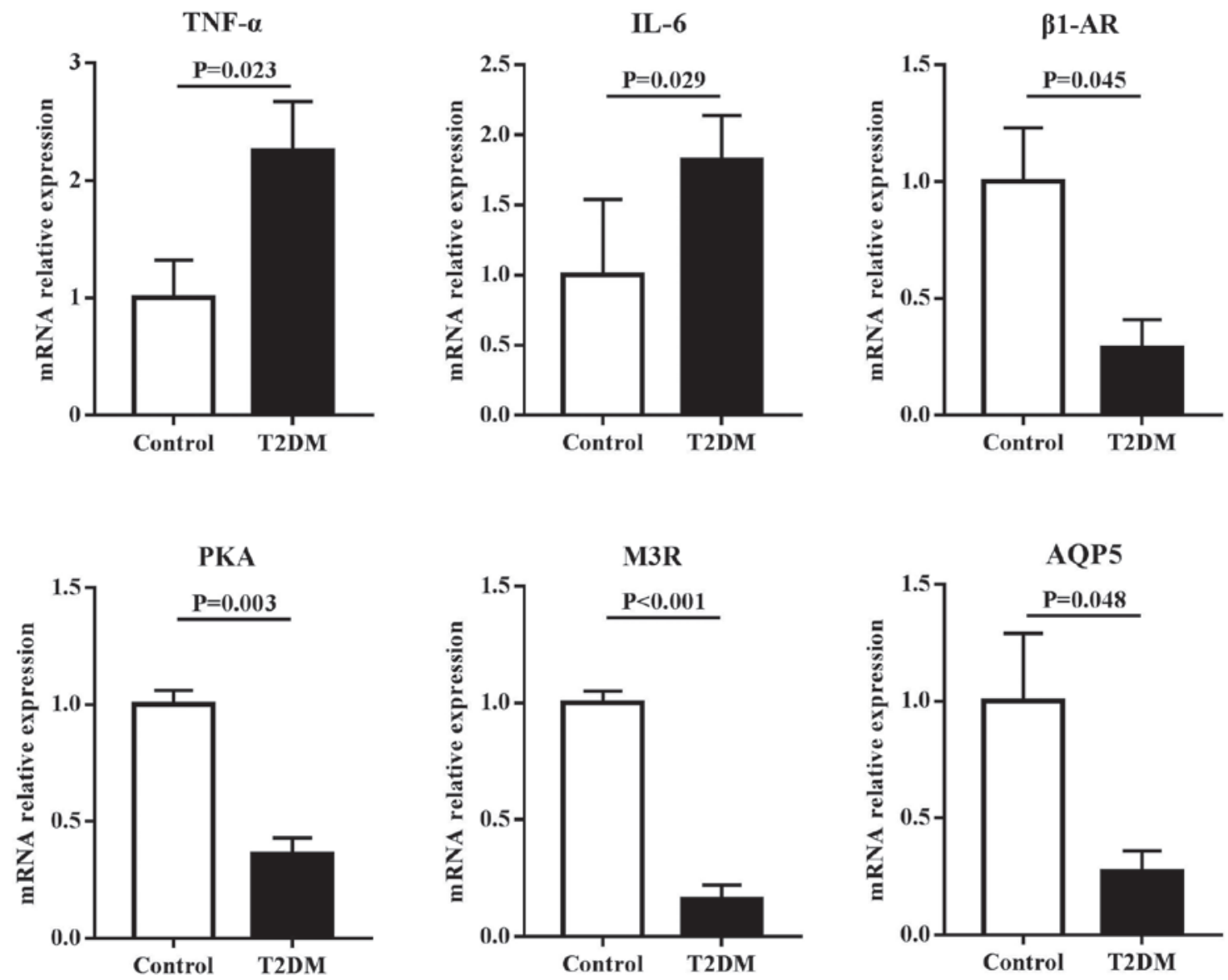

Figure 3. mRNA expression levels of inflammatory factors and regulatory proteins for salivary secretion in the parotid gland of rats. T2DM rats had significantly higher mRNA expressions of TNF- $\alpha$ and IL-6, and lower mRNA expressions of $\beta 1$-AR, PKA, M3R and AQP5 compared with control rats. T2DM, type 2 diabetes mellitus; TNF, tumor necrosis factor; IL, interleukin; $\beta 1$-AR, $\beta 1$ adrenergic receptor; PKA, protein kinase A; M3R, cholinergic receptor; AQP5, aquaporin-5.

T2DM rats, the histopathological lesions of the parotid and submandibular glands were observed in the two groups of rats by $H \& E$ staining. The $H \& E$ staining results of parotid gland were showed in Fig. 1. For control rats (Fig. 1A-a-c), the parotid lobules boundaries were clear. Normal acinar cells were observed in terms of morphology. The lumen of gland ducts was clearly visible and there were many secretory granules at the top of these gland ducts. The parietal cells of gland ducts were arranged orderly and part of gland ducts were surrounded by fibrous tissue. For T2DM rats (Fig. 1B-a-c), the parotid lobules boundaries were blurred. Acinar cell with different degrees of deformation and atrophy were observed. The cytoplasm and basal lines of acinar cells were blurred, and the vacuolization of acinar cells was obvious. The number of ducts reduced and their lumen was blurred. Moreover, reduced secretory granules at the top of gland ducts were observed, and the parietal cells of gland ducts were arranged disorderly. In addition, some ducts were dilated, and surrounded by inflammatory cell infiltration and fibrous tissue hyperplasia.

The H\&E staining results of submandibular gland were shown in Fig. 2. For control rats, serous acinar cell (Fig. 2A-a) and mucous acinar cell (Fig. 2A-b) were regular in shape, uniformly arranged and distinct in interval. Their interlobular septum was obvious, the lumen of the glandular duct was clear, and the parietal cells were arranged neatly, surrounded by fibrous tissue (Fig. 2A-c). For T2DM rats, the septum of serous acinar cells disappeared and formed cell adhesion, and the cytoplasm and basal stria of acinar cells were blurred (Fig. 2B-a). No histopathological lesions were observed in glandular ducts and mucous acinar cells (Fig. 2B-b and -c).

mRNA expression of candidate genes in salivary glands. To assess the roles of inflammation and regulatory proteins in disruption of salivary secretion in T2DM rats, the mRNA expressions of TNF- $\alpha$, IL-6, $\beta 1-A R$, M3R, AQP5 and PKA were examined in the parotid and submandibular glands. The results (Fig. 3) showed that in the parotid gland, the mRNA expression levels of inflammatory factors, including TNF- $\alpha$ and IL-6, in T2DM rats were significantly higher compared with control rats $(\mathrm{P}<0.05)$. However, the mRNA expression levels of regulatory proteins for salivary secretion, including $\beta 1-A R$, M3R, AQP5 and PKA in T2DM rats, were significantly lower compared with control rats $(\mathrm{P}<0.05)$. Similar results were found in the submandibular gland. However, no differential expression in TNF- $\alpha$ and M3R were observed between T2DM and control rats (Fig. 4).

Protein expression in salivary glands. Due to the fact that secretion of sAA in type 2 diabetic rats was more obvious, the focus of the present study was the proteins associated with 

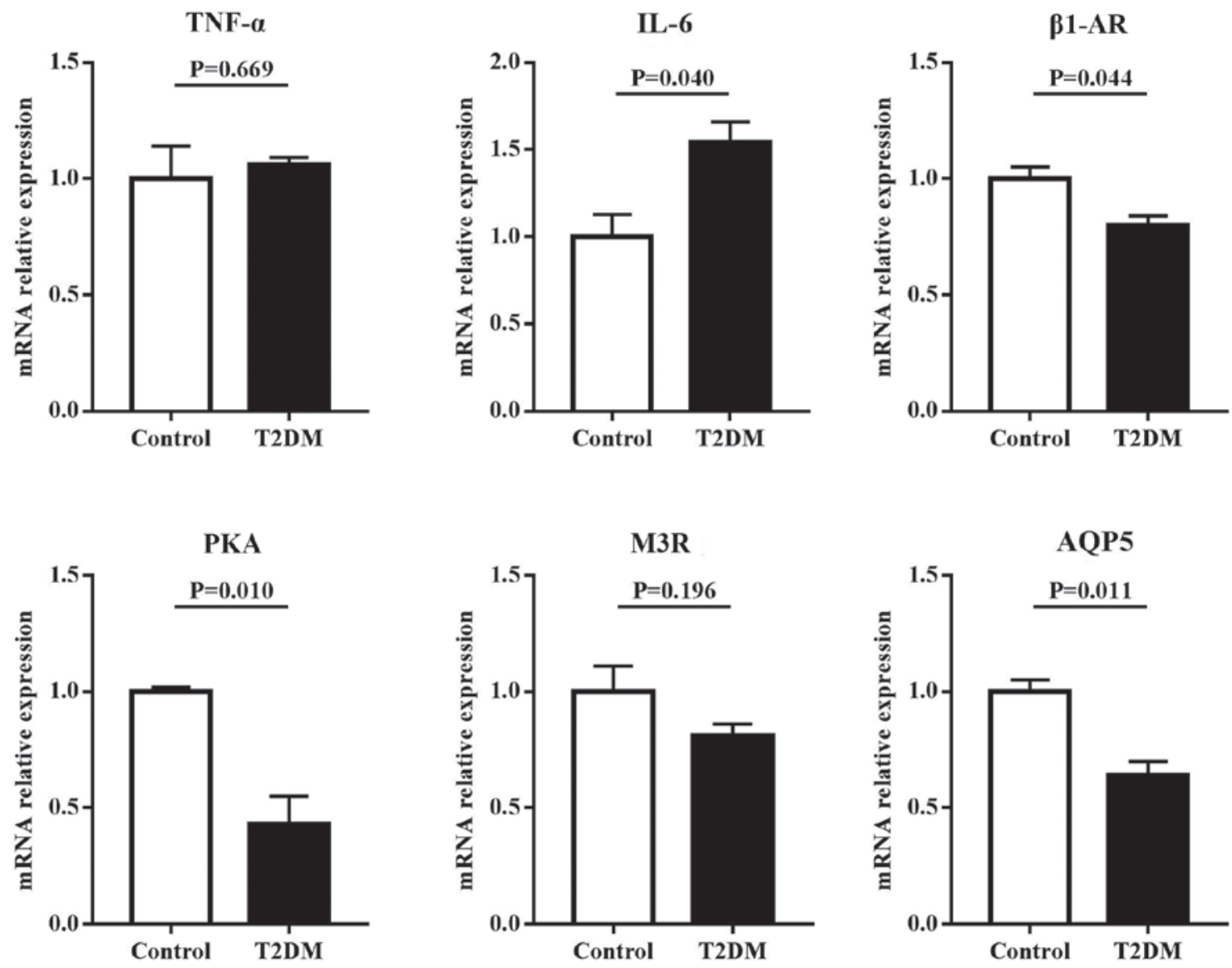

Figure 4. mRNA expression levels of inflammatory factors and regulatory proteins for salivary secretion in the submandibular gland of rats. T2DM rats had significantly higher mRNA expressions of IL-6, and lower mRNA expressions of $\beta 1$-AR, PKA and AQP5 compared with control rats. T2DM, type 2 diabetes mellitus; TNF, tumor necrosis factor; IL, interleukin; $\beta 1$-AR, $\beta 1$ adrenergic receptor; PKA, protein kinase A; M3R, cholinergic receptor; AQP5, aquaporin-5.

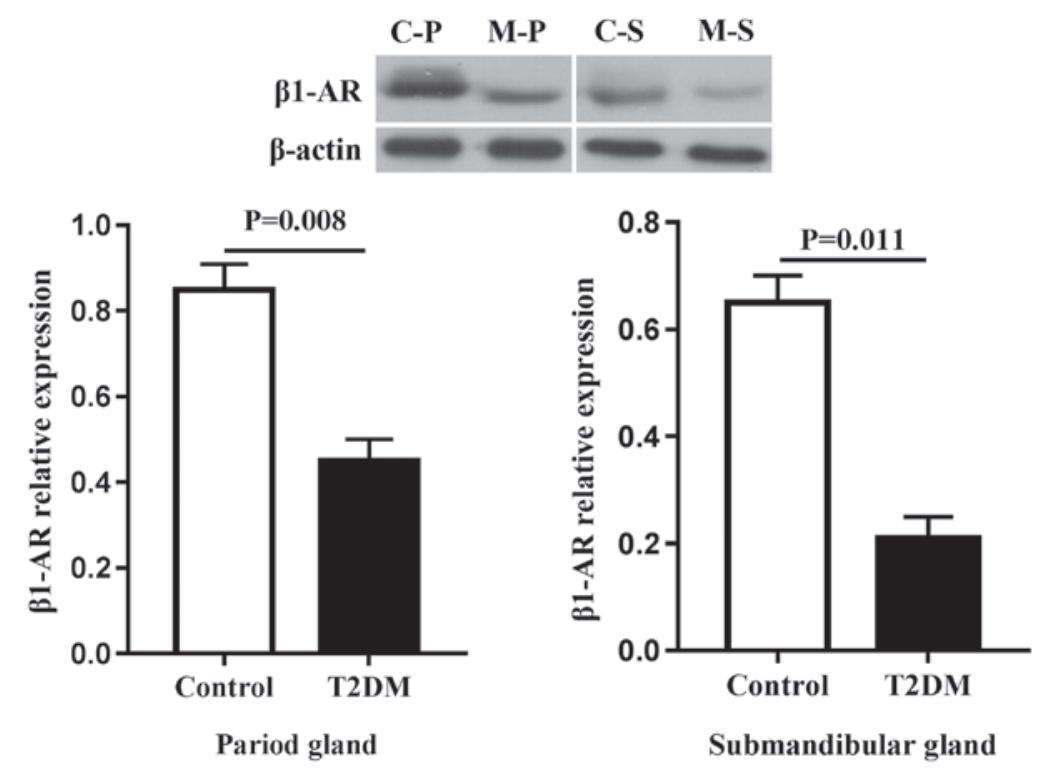

Figure 5. Protein expression of $\beta 1$-AR in salivary glands of rats. Due to the difference of expression abundance, the interest and control proteins were run on different membranes. C, control; M, T2DM; P, parotid gland; S, submandibular gland; $\beta 1-\mathrm{AR}, \beta 1$ adrenergic receptor.

sAA secretion (Figs. 5 and 6). Compared with the control group, the expression of $\beta 1-\mathrm{AR}$ and sAA decreased regardless of the types of gland, including the parotid and submandibular glands $(\mathrm{P}<0.05)$.

\section{Discussion}

In the present study, it was indicated that T2DM rats had decreased salivary secretion parameters and attenuated 

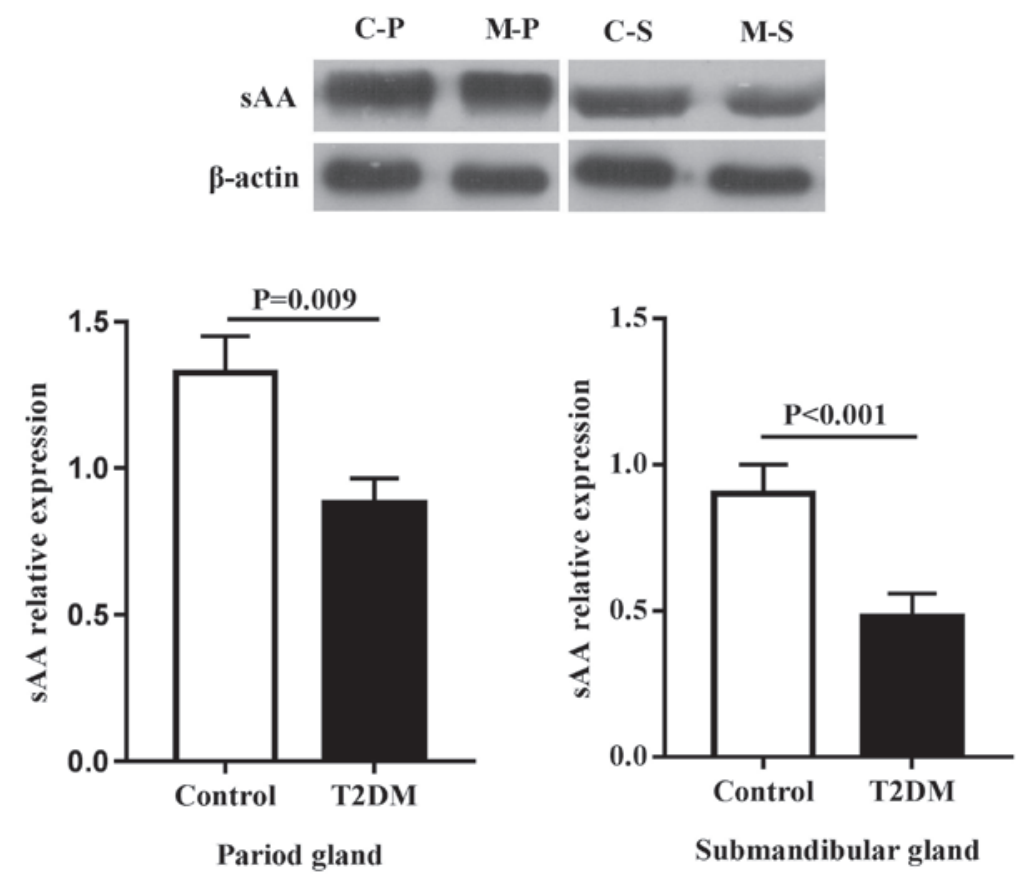

Figure 6. Protein expression of sAA in salivary glands of rats. Due to the difference of expression abundance, the interest and control proteins were run on different membranes. C, control; M, T2DM; P, parotid gland; S, submandibular gland; sAA, salivary alpha amylase.

salivary secretion responses to acid stimulation. Furthermore, morphological and structural abnormalities were observed, in addition to secretory dysfunction in salivary glands of T2DM rats, especially in the parotid gland.

Saliva includes two parts of fluid and protein saliva. The fluid saliva is mainly water and electrolyte, which is responsible for lubricating oral mucosa, dissolving food and facilitating swallowing. The protein saliva contains amylase and lysozyme, which may serve an important role in starch digestion and oral healthy. As the main component of salivary proteins, sAA was often used as a major indicator of salivary protein secretion. Recent studies have reported that sAA was associated with the occurrence of metabolic diseases, including obesity and diabetes $(15,16)$. In our previous study on salivary secretion in children, it was indicated that children with low body mass index had significantly lower sAA activity ratio, calculated as the ratio of stimulated sAA levels to those of resting sAA, compared with that of healthy children. However, no significant differences in resting or stimulated sAA activities between the two groups were observed (17). Kołodziej et al (18) found that stimulated salivary flow rate significantly decreased $(45 \%)$ in HFD-induced insulin-resistance (IR) rats compared with the control rats, however this did not apply for non-stimulated salivary flow rate. The present study found that T2DM rats had significantly lower salivary flow rate, protein secretion rate, sAA activity and sAA specific activity in basal and stimulated saliva, and attenuated salivary secretion responses to acid stimulation (decreased delta values in salivary flow rate, protein secretion rate, sAA activity and sAA specific activity), compared with control rats. Similar results have been reported in diabetic patients with decreased salivary flow rate and sAA activity in unstimulated whole saliva, and a high prevalence of oral mucosal lesions $(3-5,19)$. Despite this study's findings, a number of studies reported that unstimulated sAA activity was significantly higher in diabetics compared to healthy people $(20,21)$, and unstimulated saliva flow rates had no indicated variability between the two groups $(5,20)$. Despite existing controversy in results, patients with T2DM or animal model have indicated abnormal salivary secretion, especially sAA activity.

Salivary glands include the parotid gland, the submandibular gland and the sublingual gland, which are responsible for salivary secretion. The parotid gland serves as the main contributor of protein and fluid saliva components, which produced $\sim 80 \%$ of amylase. Under stimulated conditions, the contribution of the parotid gland increases from $20 \%$ to $>50 \%$ of total saliva (22). The present study in T2DM rats found that the parotid gland showed multiple lesions, including inflammatory cell infiltration, vacuolization and atrophy of acinar cells, and reduced secretory granules, while the submandibular gland showed only serous alveolar cell deformations and adhesion. These findings indicated that T2DM rats had structural and functional abnormalities in salivary glands, especially in the parotid gland. A previous study had reported that saliva biochemical disorders in patients with T2DM were associated with the structural changes of parotid glands (23). Xia et al (24) found that the reduction of salivary flow rate in small pigs was associated with the atrophy of the parotid gland. Teng et al (25) found that the parotid gland of T2DM rats had a number of lesions, including glandular cell hyperplasia, steatosis and interstitial hyaline degeneration. Malicka et al (26) found that high prevalence of xerostomia in diabetic patients was mostly due to impaired salivary gland function.

When examining biochemical parameters in pathological lesions of salivary glands, the present study showed that T2DM rats had lower sAA and T-SOD activities, and higher 
concentrations of blood glucose, TC and MDA in the parotid gland compared with control rats, while similar results were found in the submandibular gland, except for no difference in the two indicators of TC and MDA. In addition, the correlation analysis showed fasting glucose concentration was negatively correlated with salivary flow rate and stimulated sAA activity. Meanwhile, negative correlations between sAA activity and glucose concentration, and between T-SOD activity and total protein content in the parotid and submandibular glands was indicated. Furthermore, a positive correlation between TC and glucose concentrations in the parotid gland was found. The aforementioned findings led to the following conclusions: High sugar and fat were easier to deposit in the parotid gland, and increased TC and glucose contents decreased the synthesis and secretion of sAA in salivary glands. However, there was a higher oxidative stress in the parotid gland compared with the submandibular gland, and reduced T-SOD activity increased protein content in salivary glands. Zalewska et al (27) reported that salivary antioxidant capacity was determined mainly by the antioxidant efficiency of the parotid gland. The intensity of oxidative damage was much greater in the parotid glands of HFD-induced IR rats. This may be due to the fact that parotid gland presents adipocytes in the parenchyma, and more sensitive to combine with ROS compared with the submandibular gland. Kołodziej et al (18) also found that the vacuolization of the parotid gland appeared to be a lipid nature. In contrast to the present study's results, Kołodziej et al (18) found that HFD-induced IR rats had significantly decreased protein concentration in both glands, with a larger disruption in the parotid gland compared with the submandibular gland. However, in the present study, increased protein content was found in the submandibular gland of T2DM rats, which may be associated with the occurrence and development of T2DM. Taking the aforementioned into consideration, the pathological lesions and biochemical indicators in salivary glands can be validated by each other.

Salivary secretion is mainly controlled by the autonomic nervous system, in which the sympathetic nerve regulates the secretion of protein saliva via the $\beta$-AR pathway, and the parasympathetic nerve regulates the secretion of fluid saliva via the M3R pathway $(28,29)$. This study's results showed that mRNA expression levels of $\beta 1-A R$ and M3R were significantly down-regulated in the parotid gland of T2DM rats, while only the expression level of $\beta 1-A R$ was down-regulated in the submandibular gland of T2DM rats. In addition, the protein expression level of $\beta 1$-AR was also down-regulated in both glands. These findings suggested that there was an autonomic dysfunction in T2DM rats, further verifying the decrease of salivary flow rate, protein secretion rate, sAA activity and sAA specific activity in T2DM rats. Kołodziej et al (18) found that whole salivary flow rate stimulated by pilocarpine, a muscarinic agonist, was decreased by $45 \%$ in HFD-IR rats, compared with the control group. Anderson et al (8) confirmed that diabetic rats showed abnormal response of the parotid gland to parasympathetic nerve stimulation, while autonomic neuropathy was an important factor.

In addition, AQP5, the aquaporin firstly found in salivary glands, is a downstream signaling molecule mediated by the M3R pathway. It serves an important role in fluid synthesis and transport in vivo. The present study found that mRNA expression level of AQP5 was down-regulated in the parotid and submandibular glands of T2DM rats. These results were in accordance to previous studies, in which saliva secretory volume decreased and salivary components were altered in AQP5-mutant rats and knockout mice $(30,31)$. PKA, as a member of the salivary protein secretion pathway, was also found to be down-regulated in the parotid and submandibular glands of T2DM rats. These findings provide further explanation for the reduced salivary secretion, including salivary flow and sAA activity in T2DM rats. In addition, the present study found that T2DM rats had a lower protein secretion rate, however no difference in salivary total protein content was observed. This may be associated with the simultaneous reduction of protein and fluid saliva regulated by the dysfunctional autonomic nerve system.

In addition, inflammation is an important factor affecting the function of salivary glands. The present study found that mRNA expression levels of TNF- $\alpha$ and IL- 6 in the parotid gland of T2DM rats were significantly higher than that of control rats, which verified the inflammatory cell infiltration finding in the histopathological observation of the parotid gland. Although no inflammatory cell infiltration was found, the expression level of IL-6 was significantly up-regulated in the submandibular gland of T2DM rats. These findings indicated that T2DM rats had an inflammatory reaction in salivary glands, in particular the parotid gland. Solinas and Karin (32) found that HFD-induced obese and type 2 diabetic parotid glands enhanced storage of lipid droplets, which further activated secretion of pro-inflammatory cytokines, including TNF $\alpha$, IL-6, and IL-1 $\beta$, and ROS production in adipocytes and macrophages of parotid gland.

Overall, in the case of T2DM induced by HFD, the excess fatty acid increased insulin resistance leading to glucose and fat droplet storage in salivary glands, causing inflammation. In addition, fatty acids increased the production of reactive oxygen and nitrogen species in the respiratory chain, which may lead to the development of oxidative stress and oxidative damage. Moreover, deepening inflammation would further increase the production of free radicals, and oxidative stress may also aggravate inflammatory response. Finally, due to antioxidant deficiency, oxidative stress and inflammation damaged the structure and secretory regulation pathway of salivary glands, showing reduced salivary secretion.

In summary, T2DM rats showed significant decrease in salivary flow rate, protein secretion rate, sAA activity and sAA specific activity compared with control rats, manifesting various symptoms, including thirst and polydipsia. The pathological mechanism may be associated with histopathological lesions, including the inflammatory cell infiltration, oxidative stress, and down-regulated expression of secretory regulatory genes and proteins in salivary glands, especially in the parotid gland.

\section{Acknowledgements}

Not applicable. 


\section{Funding}

This research was supported by the Natural Science Foundation of Guangdong Province (grant no. 2017A030313837), National Natural Science Foundation of China (grant no. 81102703), Science and Technology Planning Project of Guangdong Province (grant no. 2013A032500005) and the Natural Science Foundation for Fostering of Guangdong Pharmaceutical University (grant no. GYFYLH201303).

\section{Availability of data and materials}

The datasets used and/or analyzed during the present study are available from the corresponding author on reasonable request.

\section{Authors' contributions}

ZMY conceived and designed the experiments. SYC, YW and CLZ performed the experiments. ZMY, SYC, CLZ and YW analyzed and interpreted the data. ZMY and SYC drafted and revised the manuscript. All authors read and approved the final manuscript.

\section{Ethics approval and consent to participate}

The experimental protocol was established, according to internationally recognized guidelines on animal welfare, as well as local and national regulations, in accordance with the U.K. Animals (Scientific Procedures) Act and associated guidelines, the EU Directive 2010/63/EU for animal experiments. The experimental protocol was approved by the Academic Ethics Committee of Guangdong Pharmaceutical University (approval no. gdpu2016074).

\section{Patient consent for publication}

Not applicable.

\section{Competing interests}

The authors declare that they have no competing interests.

\section{References}

1. Ma X, Chen Z, Wang L, Wang G, Wang Z, Dong X, Wen B and Zhang $Z$ : The pathogenesis of diabetes mellitus by oxidative stress and inflammation: Its inhibition by berberine. Front Pharmacol 9: 782, 2018.

2. Pedersen AML, Sørensen CE, Proctor GB, Carpenter GH and Ekström J: Salivary secretion in health and disease. J Oral Rehabil 45: 730-746, 2018.

3. Bakianian Vaziri P, Vahedi M, Mortazavi H, Abdollahzadeh SH and Hajilooi M: Evaluation of salivary glucose, IgA and flow rate in diabetic patients: A case-control study. J Dent (Tehran) 7: 13-18, 2010.

4. Indira M, Chandrashekar P, Kattappagari KK, Chandra LP, Chitturi RT and BV RR: Evaluation of salivary glucose, amylase, and total protein in type 2 diabetes mellitus patients. Indian $\mathbf{J}$ Dent Res 26: 271-275, 2015.

5. Panchbhai AS, Degwekar SS and Bhowte RR: Estimation of salivary glucose, salivary amylase, salivary total protein and salivary flow rate in diabetics in India. J Oral Sci 52: 359-368, 2010.
6. Yin Z,Zhang YY and Cui LD: Clinical significance of combined detection of serum tumor necrosis factor, sialic acid and alpha-1 acid glycoprotein in type 2 diabetes mellitus. J Chin Med University 6: 78-79, 2004 (In Chinese).

7. Marín Martínez L, Molino Pagán D and López Jornet P: Trace elements in saliva as markers of type 2 diabetes mellitus. Biol Trace Elem Res 186: 354-360, 2018.

8. Anderson LC, Garrett JR, Thulin A and Proctor GB: Effects of streptozocin-induced diabetes on sympathetic and parasympathetic stimulation of parotid salivary gland function in rats. Diabetes 38: 1381-1389, 1989.

9. Stewart CR, Obi N, Epane EC, Akbari AA, Halpern L, Southerland JH and Gangula PR: The effects of diabetes on salivary gland protein expression of tetrahydrobiopterin and nitric oxide synthesis and function. J Periodontol 87: 735-741, 2016.

10. Mori Y, Muratsu K, Nara Y and Morioka T: The histopathological observation of the salivary gland in hamsters with streptozotocin induced diabetes. Fukuoka Igaku Zasshi 81: 298-302, 1990 (In Japanese).

11. High AS, Sutton J and Hopper AH: A morphometric study of submandibular salivary gland changes in streptozotocin-induced diabetic rats. Arch Oral Biol 30: 667-671, 1985.

12. Widjaja J, Dolo PR, Zhang Q, Yao L, Li C, Hong J, Wang H, Meng S, Shao Y and Zhu X: Bypassed and preserved stomach resulted in superior glucose control in sprague-dawley rats with streptozotocin-induced diabetes. Sci Rep 9: 9981, 2019.

13. Lin J, Lu Q and Yang ZM: Evaluation of saliva collection method in rat model of spleen-deficiency by using salivary alpha-amylase activity index. J Basic Chin Med 22: 909-911+924, 2016 (In Chinese).

14. Lin J, Yang ZM and Lu Q: Evaluation on the methods for collecting saliva before and after acid stimulation by salivary flow rate and sAA activity in rats. J Guangdong Pharm University 30: 753-757, 2014 (In Chinese).

15. Mandel AL and Breslin PA: High endogenous salivary amylase activity is associated with improved glycemic homeostasis following starch ingestion in adults. J Nutr 142: 853-858, 2012.

16. Elder PJD, Ramsden DB, Burnett D, Weickert MO and Barber TM: Human amylase gene copy number variation as a determinant of metabolic state. Expert Rev Endocrinol Metab 13: 193-205, 2018.

17. Chen LH, Yang ZM, Chen WW, Lin J, Zhang M, Yang XR and Zhao LB: Attenuated acute salivary $\alpha$-amylase responses to gustatory stimulation with citric acid in thin children. Br J Nutr 113: 1078-1085, 2015.

18. Kołodziej U, Maciejczyk M, Miąsko A, Matczuk J, Knaś M, Żukowski P, Żendzian-Piotrowska M, Borys J and Zalewska A: Oxidative modification in the salivary glands of high fat-diet induced insulin resistant rats. Front Physiol 8: 20, 2017.

19. Silva MF, Barbosa KG, Pereira JV, Bento PM, Godoy GP and Gomes DQ: Prevalence of oral mucosal lesions among patients with diabetes mellitus types 1 and 2. An Bras Dermatol 90: 49-53, 2015.

20. Aydin S: A comparison of ghrelin, glucose, alpha-amylase and protein levels in saliva from diabetics. J Biochem Mol Biol 40: 29-35, 2007.

21. Hamed M, Mina J, Maryam B and Hamidreza A: Salivary alpha-amylase alteration as a possible indicator for diabetes. J Basic Appl Sci Res 4: 284-288, 2014.

22. Rohleder N and Nater UM: Determinants of salivary $\alpha$-amylase in humans and methodological considerations. Psychoneuroendocrinology 34: 469-485, 2009.

23. Carda C, Mosquera-Lloreda N, Salom L, Gomez de Ferraris ME and Peydró A: Structural and functional salivary disorders in type 2 diabetic patients. Med Oral Patol Oral Cir Bucal 11: E309-E314, 2006 (In English, Spanish).

24. Xia DS, Liu Y, Zhang CM, Yang SH and Wang SL: Observation of salivary flow rate and oral bacterial changes in small pigs after bilateral parotid atrophy. Chin J Stomatol 42: 737-740, 2007 (In Chinese).

25. Teng YJ, Chen P, Zhao HT, Yang XF, Nian H and Dong DW: The effect of ginkgo biloba extract on morphological character of parotid gland and submandibular gland of diabetic rats. Acta Chin Med Pharmacol 39:21-23, 2011 (In Chinese).

26. Malicka B, Kaczmarek U and Skośkiewicz-Malinowska K: Prevalence of xerostomia and the salivary flow rate in diabetic patients. Adv Clin Exp Med 23: 225-233, 2014.

27. Zalewska A, Knaś M, Zendzian-Piotrowska M, Waszkiewicz N, Szulimowska J, Prokopiuk S, Waszkiel D and Car H: Antioxidant profile of salivary glands in high fat diet-induced insulin resistance rats. Oral Dis 20: 560-566, 2014. 
28. Proctor GB and Carpenter GH: Regulation of salivary gland function by autonomic nerves. Auton Neurosci 133: 3-18, 2007.

29. Ishikawa Y, Cho G, Yuan Z, Skowronski MT, Pan Y and Ishida $\mathrm{H}$ : Water channels and zymogen granules in salivary glands. J Pharmacol Sci 100: 495-512, 2006.

30. Murdiastuti K, Purwanti N, Karabasil MR, Li X, Yao C, Akamatsu T, Kanamori N and Hosoi K: A naturally occurring point mutation in the rat aquaporin 5 gene, influencing its protein production by and secretion of water from salivary glands. Am J Physiol Gastrointest Liver Physiol 291: G1081-G1088, 2006.
31. Ma T, Song Y, Gillespie A, Carlson EJ,Epstein CJ and Verkman AS: Defective secretion of saliva in transgenic mice lacking aquaporin-5 water channels. J Biol Chem 274: 20071-20074, 1999.

32. Solinas G and Karin M: JNK1 and IKKbeta: Molecular links between obesity and metabolic dysfunction. FASEB J 24: 2596-2611, 2010.

(i) $\odot$ This work is licensed under a Creative Commons Attribution-NonCommercial-NoDerivatives 4.0 International (CC BY-NC-ND 4.0) License. 